\title{
International Society for the Advancement of Spine Surgery Guideline-Intraosseous Ablation of the Basivertebral Nerve for the Relief of Chronic Low Back Pain
}

\author{
MORGAN LORIO, MD, FACS, ${ }^{1}$ OLIVIER CLERK-LAMALICE, MD ${ }^{2}$ DOUGLAS P. BEALL, MD, ${ }^{3}$ \\ TERRENCE JULIEN, MD, FACS ${ }^{4}$ \\ ${ }^{I}$ Advanced Orthopedics, Altamonte Springs, Florida, ${ }^{2}$ Beam Interventional \& Diagnostic Imaging, Calgary, Canada, ${ }^{3}$ Clinical Radiology of Oklahoma, Edmond, \\ Oklahoma, ${ }^{4}$ BayCare Medical Group, Tampa, Florida
}

\begin{abstract}
RATIONALE
This International Society for the Advancement of Spine Surgery guideline is generated to respond to growing requests for background, supporting literature and evidence, and proper coding for intraosseous ablation of the basivertebral nerve for chronic low back pain.
\end{abstract}

Testing \& Regulatory Affairs

Keywords: intraosseous ablation, basivertebral nerve, chronic low back pain, vertebrogenic pain

\section{INTRODUCTION}

\section{Prevalence and Clinical Presentation}

Low back pain (LBP) is the most expensive occupational disorder in the United States and the leading cause of disability worldwide. ${ }^{1-3}$ Thirty percent of Americans have LBP at any given time, leading to approximately 50 million physician visits in the US annually. Although many of these patients improve with little to no treatment, an estimated 30 million adults in the US currently suffer from chronic LBP (CLBP), defined as pain lasting for greater than 12 weeks. $^{4-10}$ These CLBP patients have direct yearly costs of over $\$ 90$ billion/year. ${ }^{11}$ As is the case with many medical conditions, a minority of CLBP patients consume the majority of health care resources. Analyses of commercial payer and Medicare claims databases reveals that $15 \%$ of CLBP patients account for $75 \%$ of health care costs, with average claims of \$24 700 over a 3-year period in the high health care use group (MarketScan, Truven Health Analytics from October 2011 to September 2016).

Disc degeneration (DD) is a strong risk factor for CLBP,${ }^{12-14}$ and the disc has been the target of many treatments. Recent scientific research has reexamined CLBP sources, and there is evidence suggesting that the disc and adjacent endplates act as 1 functional unit and that the vertebral endplate is a source of pathologic innervation that occurs with DD.
Indeed, the endplates must balance conflicting requirements of being strong to prevent vertebral fracture and being porous to facilitate transport between disc cells and vertebral capillaries. Consequently, endplates are particularly susceptible to damage leading to inflammation and nerve proliferation.

The sensory nerves within the center of the vertebral body converge to form the basivertebral nerve (BVN). ${ }^{15,16}$ The BVN exits the vertebral body posteriorly via the basivertebral foramen before communicating with the sinuvertebral nerve then the ventral rami of the spinal nerves or by nerves derived from the gray rami communicantes ${ }^{16}$ When the density of pain fibers between normal endplates and degenerated endplates is compared, the BVN density is considerably higher in patients with degenerated endplates, further suggesting the role of pain transmission via the BVN in patients with CLBP. ${ }^{16}$ The pain transmission of the endplates toward the BVN has been named of "vertebrogenic" origin. ${ }^{14,15}$ Patients with vertebrogenic pain are thought to present with LBP, with or without referral into the buttocks or thighs (somatic referred pain).

\section{Traditional Treatments for CLBP}

CLBP may lead to a compromised quality of life, strained societal and familial relationships, and increased absenteeism or work-related disability 
Table 1. Nonsurgical management often used for chronic low back pain.

1. Avoidance of activities that aggravate pain

2 .Trial of chiropractic manipulation

3. Trial of physical therapy

4. Cognitive support and recovery reassurance

5. Spine biomechanics education

6. Specific lumbar exercise program

7. Home use of heat/cold modalities

8. Low-impact aerobic exercise as tolerated

9. Pharmacotherapy (eg, nonnarcotic analgesics, nonsteroidal antiinflammatory drugs)

claims. A lack of current validated diagnostic reference standards leads $85 \%$ of individuals to be diagnosed with nonspecific LBP. This nonspecific diagnosis leads to nonspecific care that follows care pathways that are not scientifically validated (Table 1). Individuals are advised to stay active, engage in core strengthening, lose weight, and avoid bed rest. They are put through nonsequential, palliative injection treatments in the hope that these treatments will help the patient's function and that the pain will then regress. For refractory cases, surgical intervention may be recommended.

\section{Radiologic Imaging}

The magnetic resonance imaging (MRI) correlation between vertebral endplate pathology and CLBP was made by Modic et $\mathrm{al}^{17}$ in 1988 , who described intraosseous MRI changes adjacent to damaged vertebral endplates in individuals with CLBP.

This correlation is based on the T1- and T2weighted signal of the endplates. Three types of signal change have been described: Modic change 1 (MC1), Modic change 2 (MC2), and Modic change 3 (MC3). MC1 corresponds to bone marrow edema and inflammation (hypointense T1-weighted signal and hyperintense T2-weighted signal; Figures 1A and $1 \mathrm{~B}) . \mathrm{MC} 2$ is characterized by hyperintense T1weighted signal and hyperintense T2-weighted signal (Figures 1C and 1D) and is the conversion of normal red hemopoietic bone marrow into yellow fatty marrow. MC3 is described as bone sclerosis and is characterized by hypointense T1-weighted signal and hypointense T2-weighted signal. MC1 is considered unstable, and some studies have suggested this to be painful. ${ }^{18,19} \mathrm{MC} 2$ has been suggested to be less correlated to pain. ${ }^{20}$ Patients with MC3 change are rarely symptomatic. Some speculate that the $\mathrm{MC1}$ change is caused by an inflammatory response due to fissuring and disruption between the disc and the bone that develops along with endplate microfractures, ${ }^{18-22}$ while others think that some of these changes could be due to a chronic infection. ${ }^{23}$

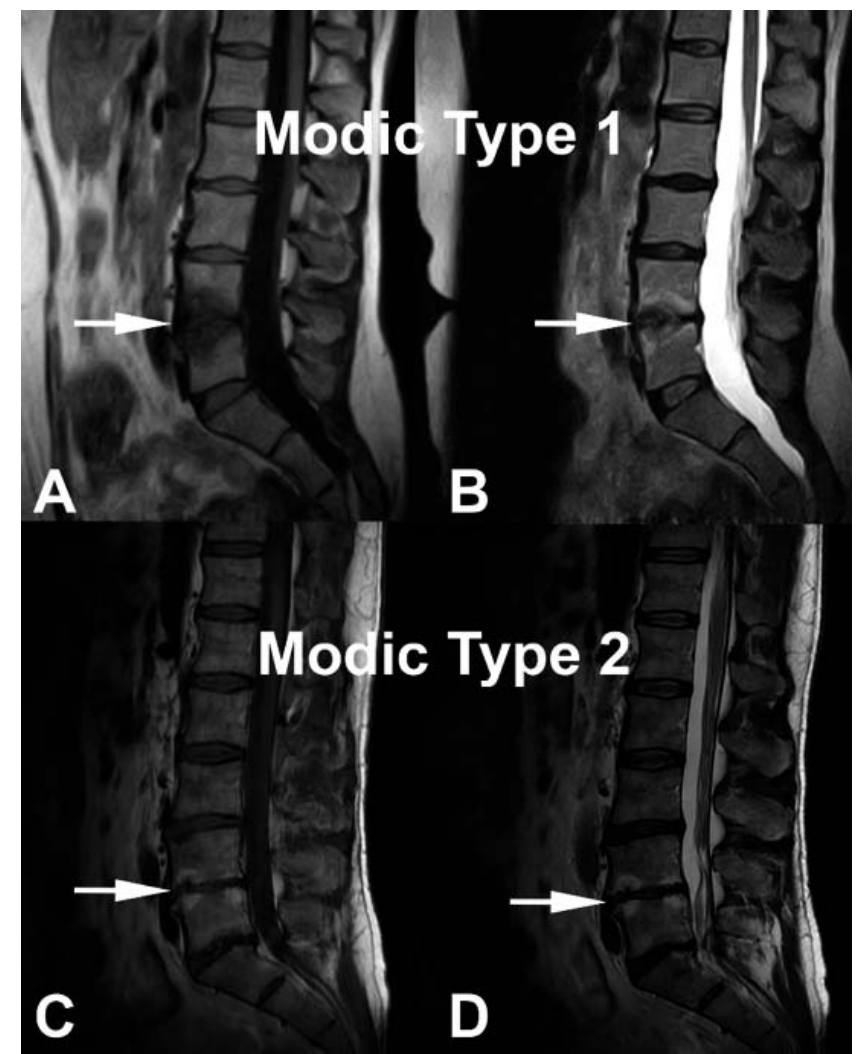

Figure 1. Modic change 1 (MC1) and Modic change 2 (MC2) illustrated. (A) and $(B)$ demonstrate decreased signal intensity on T1-wighted images and increased signal intensity on T2-weighted images, respectively (white arrows) corresponding to MC1. (C) and (D) correspond to MC2 with increased signal intensity on T1-weighted images and on T2-weighted images, respectively (white arrows).

The afferent pain pathway travels from the disc and endplate to converge as the BVN before being transmitted through the dorsal root ganglion to the central nervous system and perceived as LBP. The initial neural convergence at the BVN in the midportion of the vertebral body provides a potential target for treatment. Having an MRI done prior to the patient's consultation with CLBP is essential to adequately determine the pain generator and viable treatment alternatives. Painful Modic changes most frequently affect the L4-L5 and L5-S1 levels; in fact, Kuisma et $\mathrm{al}^{24}$ found a 2.28 odds ratio for the presence of Modic changes at L5-S1 in individuals with CLBP.

\section{Procedure}

A unilateral transpedicular approach is used to advance a straight introducer under fluoroscopic guidance to the juncture of the pedicle and the vertebral body. A curved cannula assembly is used to penetrate the vertebral body and navigate toward the BVN, which is located in the posterior half of 
the vertebral body. A straight channeling stylet is then used to extend the channel to the midline location of the BVN. A bipolar probe is inserted into the posterior half of the vertebral body, connected to the radiofrequency (RF) generator, and energy is applied for 15 minutes to destroy the BVN. Once ablated, these nerves no longer transmit pain signals.

Data from the 2 level 1 randomized controlled trials (RCTs) would suggest that, in approximately $80 \%$ of patients, 2 vertebral bodies are treated, which constitute 1 vertebral motion segment. In the remaining patients, 1 or 2 additional vertebral bodies are treated for a total of 2-3 vertebral motion segments.

Animal studies performed as a part of a Food and Drug Administration (FDA) submission also showed that the intraosseous BVN does not regenerate and that the vertebrae return to pretreatment strength after a period of normal healing (written communication, Professor Jeffrey C. Lotz, $\mathrm{PhD}$ [David S. Bradford, MD Endowed Chair in Orthopaedic Surgery at UCSF] and corroborated by published bovine research by Hoopes et $\mathrm{al}^{25}$ ).

\section{PUBLISHED LITERATURE}

\section{Becker et $\mathrm{al}^{26}$ Pilot Study}

Single-arm, open-label, first-in-human pilot study to determine the early efficacy and safety of intraosseous BVN ablation for the treatment of CLBP. Seventeen patients with 6 or more months of CLBP and $\mathrm{MC1}$ or $\mathrm{MC} 2$ changes were enrolled. Sixteen patients were successfully treated using RF energy to ablate the BVN within the vertebral bodies adjacent to the diagnosed level (based on positive discography).

The mean age of enrolled patients was 48 years. Baseline measurements of mean Oswestry Disability Index (ODI) and visual analog scale (VAS) were $52 \pm 13$ (severe disability on the 0 - to 100-point disability impact scale) and 61 (on the 0- to 100-point LBP scale). Statistically significant improvements were noted in all outcome measures at 3 months. ODI decreased an average of 29 points to a mean of $23 \pm 21$ at 3 months of follow up $(P<.001)$. This statistically significant improvement in ODI was maintained through the 12 month follow up.

\section{Truumees et $\mathrm{al}^{27}$ Case Series}

This study was a prospective, single-arm, multicenter, open-label study to evaluate the effectiveness of intraosseous RF ablation of the BVN for the treatment of presumed vertebrogenic-related CLBP in typical spine practice settings with more permissive inclusion of typical CLBP patients (such as patients who have had prior discectomy and users of extended-release narcotics). Consecutive patients with CLBP of at least 6 months duration and with $\mathrm{MC} 1$ or MC2 vertebral endplate changes between L3 to S1, were treated with RF ablation of the BVN in up to 4 vertebral bodies. The primary endpoint was patient-reported change in ODI from the baseline to 3 months postprocedure. Secondary outcome measures included change in LBP pain VAS, Short Form 36 (SF-36), EQ-5D-5L, and responder rates.

The median age of patients was 45 years within the 28 patients enrolled. The baseline ODI was 48.5 and VAS was $6.36 \mathrm{~cm}$ (on a 0 to $10 \mathrm{~cm}$ scale). Seventy-five percent of the study patients reported LBP symptoms for $\geq 5$ years with $25 \%$ actively using opioids and $61 \%$ previously treated with injections. Clinically meaningful and statistically significant improvements were demonstrated in all outcome measures at the 3 month primary endpoint. Mean reduction in ODI from the baseline at 3 months posttreatment was $-30.07+14.52$ points $(P<.0001)$. The mean reduction in VAS pain score from the baseline was $-3.50+2.33(P<.0001)$. Using a minimal clinically important difference (MCID) of $\geq 10$-point improvement in ODI, 93\% of patients were responders; using MCID of a $\geq 20$ point improvement in ODI, $75 \%$ were responders. Likewise, VAS MCID of a $\geq 2.0 \mathrm{~cm}$ reduction was achieved in $75 \%$ of patients. Importantly, in this population of working-aged individuals, $83 \%$ reported improvement in work function. This nonrandomized consecutive series study demonstrated that minimally invasive RF ablation of the BVN resulted in a significant improvement in pain and function at 3 months in this population of realworld patients with chronic suspected vertebrogenic related $\mathrm{LBP}$.

\section{INTRACEPT Study ${ }^{28}$}

This prospective, parallel, open-label, randomized control trial conducted at 20 US sites compared the effectiveness of intraosseous RF ablation of the BVN with standard care for the treatment of CLBP in patients suspected to have vertebrogenic-related pain symptomatology. A total of 140 patients with CLBP of at least 6 months duration, with MC1 or 


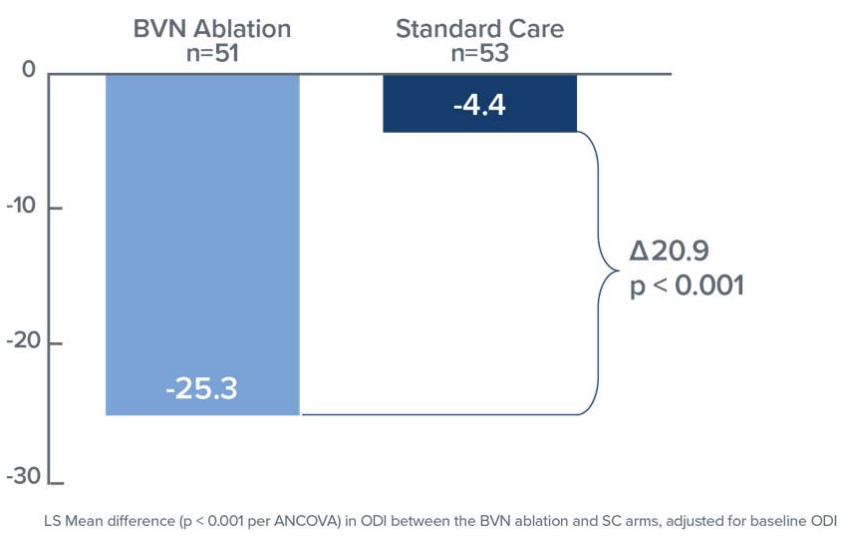

Figure 2. Bar graph demonstrating mean Oswestry Disability Index changes at 3 months both for the basivertebral nerve (BVN) ablation and the standard care groups. Statistically significant improvement of the patients' function is noted in the BVN group $(P<.001)$.

MC2 vertebral endplate changes between L3 to S1, were randomized 1:1 to undergo either RF ablation of the BVN or continue standard care. The primary endpoint was a between-arms comparison of the mean change in ODI from the baseline to 3 months posttreatment. Secondary outcome measures included LBP pain scores via VAS, ODI, VAS responder rates, SF-36, and EQ-5D-5L at 3, 6, 9, and 12 months postprocedure. An interim analysis to assess for superiority was prespecified and overseen by an independent data management committee (DMC) when a minimum of $60 \%$ of patients had completed their 3 month primary endpoint visit.

The interim analysis showed clear statistical superiority $(P<.001)$ for all primary and secondary patient-reported outcome measures in the RF ablation arm compared with ongoing standard care arm. This resulted in a DMC recommendation to halt enrollment in the study and offer early crossover to the control arm. As a result, the study reported the outcomes of the 104 patients included in the intent-to-treat analysis of the 3 month primary endpoint, which included 51 patients in the RF ablation arm and 53 patients in the standard care arm. At the baseline, the mean age was 50 years, mean ODI was 46.1 (severe pain disability), and mean VAS was $6.67 \mathrm{~cm}$ (on a 0 to $10 \mathrm{~cm}$ scale). More than $67 \%$ of patients reported experiencing LBP for greater than 5 years, and more than $70 \%$ had received prior injections at the baseline.

Comparing the RF ablation arm with the standard care arm (Figure 2), the mean changes in ODI at 3 months were -25.3 points versus -4.4 points, respectively, resulting in an adjusted difference of 20.9 points $(P<.001)$; and mean changes in

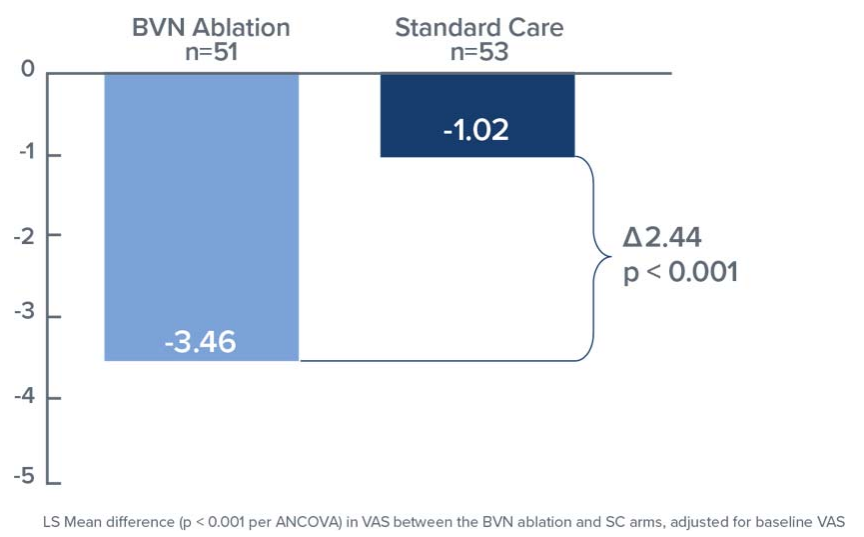

Figure 3. Bar graph demonstrating the mean difference in the visual analog scale at 3 months both for the basivertebral nerve (BVN) ablation and standard care groups. Statistically significant improvement in patients treated with BVN is noted $(P<.001)$

VAS were -3.46 versus -1.02 , respectively, an adjusted difference of $2.44 \mathrm{~cm}(P<.001$; Figure $3)$. In the RF ablation arm, $74.5 \%$ of patients achieved the MCID of $\geq 10$-point improvement in ODI, compared with $32.7 \%$ in the standard care arm $(P<.001)$. With a MCID of $2.0 \mathrm{~cm}$ improvement in VAS, $72.5 \%$ of patients in the RF ablation arm reached clinical success compared with $34.0 \%$ of patients in the standard care arm. No RF ablation patients received a spinal injection prior to the 3 month endpoint, while in the standard care arm, 6 standard of care patients $(11 \%)$ received injections across 5 study sites. The study concluded that minimally invasive RF ablation of the BVN leads to significant improvement of pain and function at 3 months in patients with suspected chronic vertebrogenic related LBP.

\section{SMART Trial ${ }^{29}$}

The SMART trial was a prospective randomized, sham-controlled, double-blinded, FDA-Investigational Device Exemption trial conducted to evaluate the safety and efficacy of RF ablation of the BVN for the treatment of CLBP. A total of 225 CLBP patients with $\mathrm{MC1}$ or $\mathrm{MC2}$ noted in vertebral bodies L3 to S1 were randomized to either a shamcontrol (78 patients) or BVN ablation treatment (147 patients). All study participants were treated with the same operating protocol and pedicle access. The sham-control arm received simulated RF ablation therapy. Treatment success was adjudicated in a blinded review of the 6-week MRI. Study participants were followed at 2 and 6 weeks and 3, 6, 9 , and 12 months postrandomized intervention. The primary efficacy endpoint was change in ODI from 
the baseline to 3 months postprocedure. The primary safety endpoint was a comparison of musculoskeletal and neurologic adverse events at 12 months.

Participants in this study were of working age (mean of 47 years), reported severe disability impact from their LBP (mean ODI of 42), and more than $68 \%$ had been experiencing CLBP for greater than 5 years. At 3 months, the mean ODI in the treatment arm decreased 20.5 points, as compared with a 15.2 point decrease in the sham arm $(P=.019$, perprotocol population). The reduction in ODI experienced by the treatment arm was twice the MCID of $\geq 10$ points and responder rates were $75.6 \%$ in the treatment arm compared with $55.3 \%$ in the shamcontrol arm. There were no serious device- or procedure-related adverse events reported in patients randomized to the RF ablation treatment arm through 12 months.

This level 1 trial demonstrated significant functional improvement in patients treated with $\mathrm{RF}$ ablation of the BVN for CLBP compared with patients treated with a sham procedure. Safety of the procedure was also demonstrated. The results supported BVN ablation as a minimally invasive treatment for the relief of CLBP.

\section{SMART 24 Month Outcomes ${ }^{30}$}

This prospective, single-arm study is an extension of follow up for the RF ablation treatment arm of the SMART trial. Per the original SMART RCT protocol, at completion of the 12 month primary safety endpoint, patients in the sham-control arm could cross to BVN ablation treatment; $73 \%$ elected to cross. Due to this high rate of crossover, the 147 RF ablation treatment arm participants acted as their own control in comparing 24 month outcomes with the baseline.

Clinical improvements in the ODI, VAS, and the SF-36 physical component summary (PCS) were statistically significant compared with the baseline at all follow-up timepoints through 2 years $(3,6,9$, 12,18 , and 24 months). The mean percent improvements at 2 years in ODI (Figure 4) and VAS compared with the baseline were $53.7 \%$ and $52.9 \%$, respectively. Responder rates for ODI and VAS were also maintained through 2 years for both a 10-point ODI MCID threshold $(76.4 \%$ of patients) and an ODI 20-point improvement threshold $(57.5 \%$ of patients); the MCID threshold for VAS of $1.5 \mathrm{~cm}$ improvement was reported in $70.2 \%$

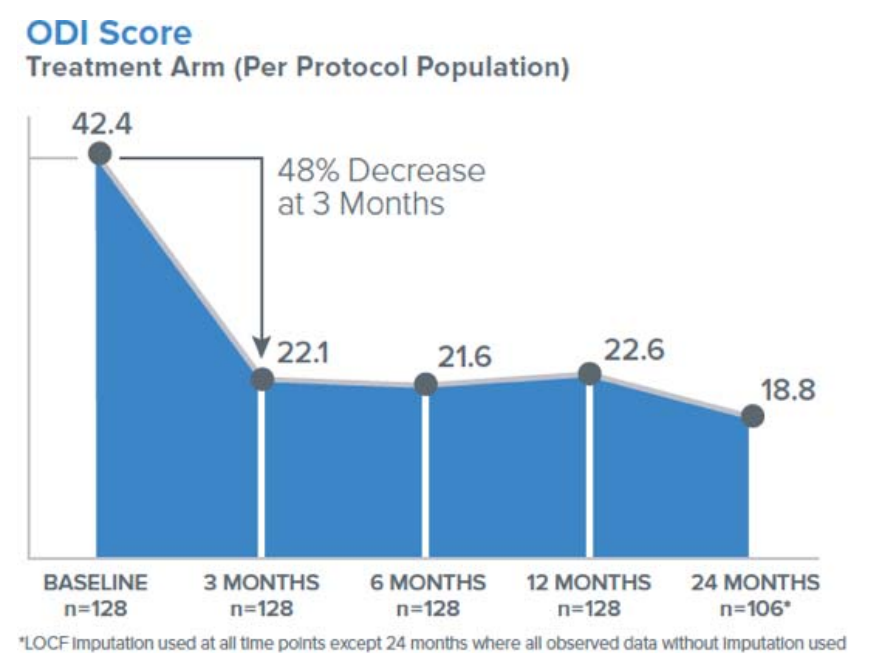

Figure 4. Mean Oswestry Disability Index score in per-protocol treatment arm followed up to 24 months.

of patients at 24 months (Figure 5). In summary, patients treated with RF ablation of the BVN for CLBP exhibited sustained clinical benefits in ODI and VAS and maintained high responder rates through 2 years following treatment.

\section{Evidence and Literature Conclusion}

Intraosseous ablation of the BVN is supported by a basic and clinical evidence foundation, including a level 1, sham-controlled RCT and a second level 1 RCT against standard conservative management. Data through 24 months suggest durability of the treatment effect. Collectively, these studies demonstrate that BVN ablation provides clinically meaningful improvements in pain and function to 2 years with an excellent safety profile. This evidence supports BVN as a treatment option for a welldefined subpopulation of CLBP patients.

\section{INDICATIONS FOR SURGERY}

Intraosseous ablation of the BVN from the L3 through S1 vertebrae may be considered medically

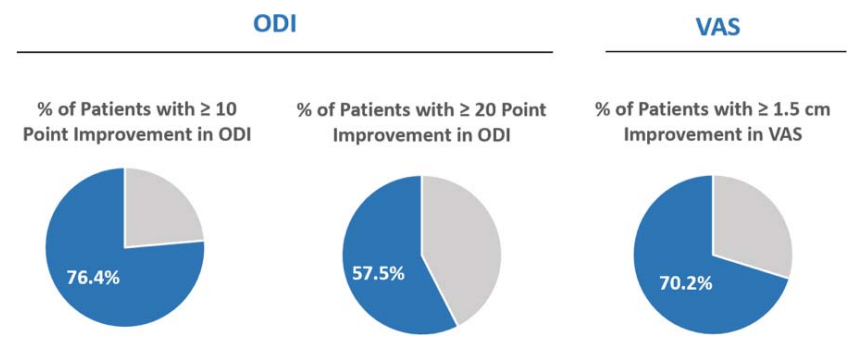

Figure 5. Shows the 24 month Oswestry Disability Index and visual analog scale responder rate per-protocol arm at 24 months $(\mathrm{N}=106)$. 
indicated for individuals with CLBP when all the following criteria are met:

- CLBP of at least 6 months duration,

- Failure to respond to at least 6 months of nonsurgical management, and

- MRI-demonstrated MC1 or MC2 in at least 1 vertebral endplate at 1 or more levels from L3 to S1.

\section{CODING AND COVERAGE HISTORY}

Intraosseous ablation of the $\mathrm{BVN}$ is a new procedure not previously performed. As such, this procedure currently should be reported with Current Procedural Terminology 22899 (unlisted procedure, spine).

Typical International Classification of Diseases, 10th Revision diagnosis codes that indicate medical necessity are as follows:

- M47.816: Spondylosis without myelopathy or radiculopathy, lumbar region,

- M47.817: Spondylosis without myelopathy or radiculopathy, lumbosacral region,

- M51.36: Other intervertebral DD, lumbar,

- M51.37: Other intervertebral DD, lumbosacral,

- M54.5: LBP.

Corresponding Healthcare Common Procedure Coding System codes, effective January 1, 2019, are as follows:

- C9752: Destruction of intraosseous BVN, first 2 vertebral bodies, including imaging guidance (eg, fluoroscopy), lumbar/sacrum,

- C9753: Destruction of intraosseous BVN, each additional vertebral body, including imaging guidance (eg, fluoroscopy), lumbar/ sacrum (list separately in addition to code for primary procedure.

\section{CONCLUSIONS}

Intraosseous ablation of the BVN is a relatively new minimally invasive treatment for the relief of CLBP that is diagnosed using well-established clinical and MRI findings. The procedure is supported by level 1 evidence including 2 RCTs demonstrating a statistically significant decrease in pain and an improvement in function with outcomes sustained to at least 24 months in a limited number of studies. These results were seen in a patient population that is one of the most expensive and difficult to provide care for, and in this era of rising health care costs and increasing need for therapies to reduce the use of opioids, BVN ablation may provide a treatment option to fill the gap in the treatment paradigm for patients that fail nonsurgical treatment.

\section{LIMITATIONS}

(1) Industry funding is a potential source of study bias for the available data reviewed.

(2) Limited number of studies.

(3) Short-term follow up for the majority of studied patients.

(4) Unknown effect on the primary degenerative process.

\section{REFERENCES}

1. Deyo RA, Mirza SK, Martin BI. Back pain prevalence and visit rates: estimates from U.S. national surveys, 2002. Spine (Phila Pa 1976). 2006;31:2724-2727.

2. Frymoyer JW. Back pain and sciatica. $N$ Engl J Med. 1988;318:291-300.

3. Hoy D, Bain C, Williams G, et al. A systematic review of the global prevalence of low back pain. Arthritis Rheum. 2012;64:2028-2037.

4. Thiese MS, Hegmann KT, Wood EM, et al. Prevalence of low back pain by anatomic location and intensity in an occupational population. BMC Musculoskelet Disord. 2014; 15:283.

5. Ganesan S, Acharya AS, Chauhan R, Acharya S. Prevalence and risk factors for low back pain in 1,355 young adults: a cross-sectional study. Asian Spine J. 2017;11(4):610-617.

6. Knauer SR, Freburger JK, Carey TS. Chronic low back pain among older adults: a population-based perspective. $J$ Aging Health. 2010;22:1213-1234.

7. Meucci RD, Fassa AG, Faria NM. Prevalence of chronic low back pain: systematic review. Rev Saude Publica. 2015;49:73. doi: 10.1590/S0034-8910.2015049005874.

8. Shmagel A, Foley R, Ibrahim H. Epidemiology of chronic low back pain in US adults: data from the 2009-2010 National Health and Nutrition Examination Survey. Arthritis Care Res (Hoboken). 2016;68:1688-1694.

9. Freburger JK, Holmes GM, Agans RP, et al. The rising prevalence of chronic low back pain. Arch Intern Med. 2009; 169:251-258.

10. Johannes CB, Le TK, Zhou X, et al. The prevalence of chronic pain in United States adults: results of an Internetbased survey. J Pain. 2010;11:1230-1239.

11. Dagenais S, Caro J, Haldeman S. A systematic review of low back pain cost of illness studies in the United States and internationally. Spine J. 2008;8:8-20.

12. Luoma K, Riihimaki H, Luukkonen R, et al. Low back pain in relation to lumbar disc degeneration. Spine (Phila $\mathrm{Pa}$ 1976). 2000;25:487-492. 
13. Cheung KM, Karppinen J, Chan D, et al. Prevalence and pattern of lumbar magnetic resonance imaging changes in a population study of one thousand forty-three individuals. Spine (Phila Pa 1976). 2009;34:934-940.

14. Livshits G, Popham M, Malkin I, et al. Lumbar disc degeneration and genetic factors are the main risk factors for low back pain in women: the UK Twin Spine Study. Ann Rheum Dis. 2011;70:1740-1745.

15. Brown MF, Hukkanen MV, McCarthy ID, et al. Sensory and sympathetic innervation of the vertebral endplate in patients with degenerative disc disease. J Bone Joint Surg Br. 1997;79:147-153.

16. Antonacci MD, Mody DR, Heggeness MH. Innervation of the human vertebral body: a histologic study. J Spinal Disord. 1998;11:526-531.

17. Modic MT, Steinberg PM, Ross JS, et al. Degenerative disk disease: assessment of changes in vertebral body marrow with MR imaging. Radiology. 1988;166(1 Pt 1):193-199. doi: 10. 1148/radiology.166.1.3336678.

18. Dudli S, Fields AJ, Samartzis D, et al. Pathobiology of Modic changes. Eur Spine J. 2016;25:3723-3734.

19. Lotz JC, Fields AJ, Liebenberg EC. The role of the vertebral end plate in low back pain. Global Spine $J$. 2013;03:153-164.

20. Toyone $\mathrm{T}$, Takahashi $\mathrm{K}$, Kitahara H, Yamagata $\mathrm{M}$, Murakami M, Moriya H. Vertebral bone-marrow changes in degenerative lumbar disc disease. An MRI study of 74 patients with low back pain. J Bone Joint Surg Br. 1994;76(5):757-764.

21. Ulrich JA, Liebenberg EC, Thuillier DU, et al. ISSLS prize winner: repeated disc injury causes persistent inflammation. Spine (Phila Pa 1976). 2007;32:2812-2819.

22. Dudli S, Sing DC, Hu SS, et al. ISSLS Prize in Basic Science 2017: intervertebral disc/bone marrow cross-talk with Modic changes. Eur Spine J. 2017;26:1362-1373.

23. Georgy M, Stern M, Murphy K. What is the role of the bacterium Propionibacterium acnes in type 1 Modic changes? A review of the literature. Can Assoc Radiol J. 2017;68(4):419424. doi: 10.1016/j.carj.2017.07.004.

24. Kjaer P, Korsholm L, Bendix T, et al. Modic changes and their associations with clinical findings. Eur Spine $J$. 2006;15:1312-1319.

25. Hoopes PJ, Eskey C, Attawia M, et al. Radiofrequency ablation of the basivertebral nerve as potential treatment of back pain: pathologic assessment in a bovine model. Proceedings of SPIE. 2005;5698:168-180.

26. Becker S, Hadjipavlou A, Heggeness MH. Ablation of the basivertebral nerve for treatment of back pain: a clinical study. Spine J. 2017;17:218-223.

27. Truumees E, Macadaeg K, Pena E, et al. A prospective, open-label, single-arm, multi-center study of intraosseous basivertebral nerve ablation for the treatment of chronic low back pain. Eur Spine J. 2019. doi: 10.1007/s00586-019-05995-2.

28. Khalil J, Smuck M, Koreckij T, et al. A prospective, randomized, multi-center study of intraosseous basivertebral nerve ablation for the treatment of chronic low back pain. Spine J. 2019. doi: 10.1016/j.spinee.2019.05.598.

29. Fischgrund JS, Rhyne A, Franke J, et al. Intraosseous basivertebral nerve ablation for the treatment of chronic low back pain: a prospective randomized double-blind shamcontrolled multi-center study. Eur Spine J. 2018;27(5):11461156. doi: 10.1007/s00586-018-5496-1.
30. Fischgrund JS, Rhyne A, Franke J, et al. Intraosseous basivertebral nerve ablation for the treatment of chronic low back pain: 2-year results from a prospective randomized double-blind sham-controlled multicenter study. Int J Spine Surg. 2019;13(2):110-119.

\section{Disclosures and COI: ML: None.}

OC: None.

DB:

- Medtronic -Advisory Board, Consultant, Research Funding

- Spineology-Consultant, Stock Owner

- Merit Medical-Consultant

- Lilly-Consultant

- Johnson \& Johnson-Consultant

- SpinTech-Consultant, Board Member, Research Funding

- Nocimed-Board Member

- Artio-Stockholder

- Flow Forward-Stockholder

- Imaging3-Adviser, Consultant, Stockholder

- IZI-Consultant

- Medlantis-Consultant

- Techlamed-Consultant

- Sway Inc-Stockholder

- Boston Scientific-Consultant, Speaker, Research Funding

- Peterson Enterprises - Consultant

- Nanofuse-Consultant, Advisory Board

- Medical Metrics-Consultant, Research Funding

- Radius Pharmaceuticals - Consultant, Speaker, Stockholder

- Avanos-Consultant, Speaker, Research Funding

- Relievant-Research Funding

- Stryker-Speaker, Research Funding

- Sollis Pharmaceuticals-Research Funding, Consultant

- Simplify Medical-Consultant, Research Funding

- Eleven Biotherapeutics - Stockholder

- Sophiris Bio-Stockholder

- Lenoss Medical-Consultant, Research Funding, Stockholder

- Spine BioPharma-Consultant, Research Funding, Stockholder

- Piramal-Consultant, Speaker

- ReGelTec-Consultant, Advisory Board

TJ:

- Medtronic-Consulting 
- Stryker Spine-Consulting

- Stryker Navigation-Consulting

- Camber Spine/IMSE - Consulting

- Aurora Spine-Consulting, Stock options

- Ulrich-Consulting, Surgeon's Advisory Board

- RTI Surgical-Consulting, Surgeon's Advisory board

- Spinal Elements - Consulting

- Bio Ventus Surgical-Consulting

- CoreLink-Consulting

- NovaBone-Consulting
Corresponding Author: Morgan Lorio, MD, FACS, Advanced Orthopedics, 499 E. Central Parkway, Altamonte Springs, FL 32701. Phone: (407) 960-1717; Email: mloriomd@gmail.com.

Published 29 February 2020

This manuscript is generously published free of charge by ISASS, the International Society for the Advancement of Spine Surgery. Copyright (C) 2020 ISASS. To see more or order reprints or permissions, see http://ijssurgery.com. 